\title{
Síndrome de Cushing Subclínica em Populações de Risco
}

\section{RESUMO}

Os incidentalomas de adrenais (IA) são tumores freqüentes em humanos. A síndrome de Cushing (SC) endógena é rara e os adenomas de adrenais são responsáveis por $10 \%$ dos casos de SC. A SC subclínica ocorre em IA com dinâmica do cortisol anormal e ausência de fenótipo característico de hipercortisolismo. A prevalência média de SC subclínica em IA é de $9 \%$. Dados de pequenas séries indicam que $20 \%$ dos IA desenvolvem alterações bioquímicas quando acompanhados por 10 anos. A evolução da SC subclínica parece ser benigna, raramente ocorrendo aumento da massa e evolução para a SC clinicamente manifesta. Os incidentalomas e a SC subclínica têm sido correlacionados aos componentes da síndrome metabólica, especialmente ao diabetes mellitus do tipo 2. Embora o número de pacientes avaliados ainda seja pequeno, os estudos disponíveis demonstram que o tratamento do hipercortisolismo resulta em melhor controle metabólico e da pressão arterial. Esses achados levaram alguns autores a pesquisar a presença de SC subclínica em pacientes com diagnóstico prévio de diabetes mellitus. Os estudos realizados utilizando diferentes abordagens diagnósticas mostraram que nesse grupo de pacientes a incidência de SC subclínica é maior do que na população geral. (Arq Bras Endocrinol Metab 2007;51/8:1185-1190)

Descritores: Diabetes mellitus tipo 2; Obesidade; Sobrepeso; Hipercortisolismo; Síndrome de Cushing; Síndrome de Cushing subclínica

\begin{abstract}
Subclinical Cushing's Syndrome in populations at Risk.

Based on autopsy studies, adrenal masses are among the most common tumors in humans. Endogenous Cushing's syndrome (CS) is unusual and adrenal adenomas account for $10 \%$ of all cases of CS. Patients with subclinical CS (SCS) present abnormal cortisol dynamics without obvious manifestations. The prevalence of hypercortisolism in clinically inapparent adrenal masses has been reported as $9 \%$. Data from several small series of patients indicate that fewer than $20 \%$ develop hormone overproduction when followed for up to 10 years. Follow-up of patients with subclinical CS suggests that rarely masses increase in size or progress to overt CS. Adrenal incidentalomas and subclinical CS are related to metabolic disorders, in special to type-2 diabetes. The scarce available data suggest that treatment of hypercortisolism correct the metabolic abnormalities and blood pressure. Some studies evaluating the prevalence of subclinical CS in overweight type-2 diabetes patients suggest that it is considerably higher in populations at risk than in the general population. (Arq Bras Endocrinol Metab 2007;51/8:1185-1190)
\end{abstract}

Keywords: Type 2 diabetes mellitus; Obesity; Overweight; Hypercortisolism; Cushing's syndrome; Subclinical Cushing's syndrome

\section{revisão}

\author{
Maria Silvia S. Caetano \\ LUCIO VILAR \\ ClaUdio E. KATER
}

Disciplina de Endocrinologia, Departamento de Medicina, Universidade Federal de São Paulo, UNIFESP/EPM (MSSC, CEK), São Paulo, SP e Hospital das Clínicas, Divisão de Endocrinologia, Universidade Federal de Pernambuco (LV), Recife, PE.

Recebido em 20/10/07 Revisado em 27/10/07 Aceito em 30/10/07 
$\mathrm{O}$ INCIDENTALOMAS DE ADRENAIS (IA) são tumores com diâmetro maior do que $1 \mathrm{~cm}$, diagnosticados casualmente em exames de imagem, na ausência de achados clínicos sugestivos de doença adrenal. Ocorrem predominantemente entre os 50 e os 70 anos. Séries de autópsias colocam os tumores de adrenais como os mais freqüentes tumores em humanos (1). A prevalência de IA em autópsias varia de 1,4 a $8,7 \%$, ocorrendo em média em $2,3 \%$ dos casos (2). Entre 82.483 tomografias avaliadas, a prevalência média foi de 0,6\% com uma variação de 0,4-9\%.

A síndrome de Cushing (SC) endógena é rara e os adenomas de adrenais são responsáveis por apenas $10 \%$ do total de casos de SC $(3,4)$. No IA a SC subclínica ocorre com dinâmica do cortisol anormal e ausência de fenótipo característico de hipercortisolismo. Assim como na SC clássica, a avaliação laboratorial na SC subclínica é realizada através de etapas e métodos que são variáveis entre diferentes centros (57). Nesses pacientes, as alterações bioquímicas do eixo hipotálamo-hipófise-adrenal (HHA) são intermediárias entre os indivíduos normais e aqueles com a SC clássica (figura 1), e as alterações do ritmo e da supressão do cortisol ocorrem com maior freqüência do que a elevação de sua taxa de secreção e de excreção urinária $(8,9)$. Além da ausência de sinais de excesso hormonal, são necessárias pelo menos duas alterações do eixo HHA para o diagnóstico de SC subclínica (8).

Uma relação direta entre o diâmetro dos tumores adrenais e o seu maior potencial de malignidade já foi estabelecida (2). O carcinoma adrenocortical é encontrado em $2 \%$ dos tumores com até $4 \mathrm{~cm}$, em $6 \%$ dos tumores com 4,1-6 cm, e em até $25 \%$ dos tumores maiores do que $6 \mathrm{~cm}(1)$.

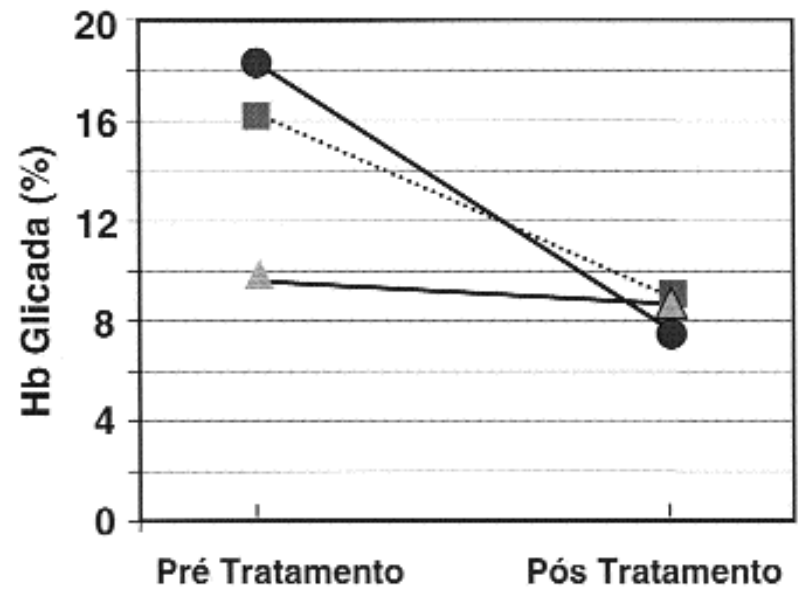

Figura 1. Evolução da hemoglobina $(\mathrm{Hb})$ glicada em três pacientes com DM, antes e após o tratamento da SC no estudo de Leibowitz et al (30).
Os adenomas, feocromocitomas, carcinomas e as metástases são os tumores mais freqüentes entre os IA (10). Entre os adenomas, os tumores "não produtores" representam a maioria, mas aproximadamente $25 \%$ são produtores de cortisol, sendo esta, sem dúvida, a hiperfunção que mais ocorre entre os incidentalomas. A prevalência média de SC subclínica em IA é de $9 \%$, com uma grande variação de 1 a 47\%, dependendo da metodologia empregada para o diagnóstico do hipercortisolismo.

O tamanho também é relacionado à hiperfunção e, embora tumores menores do que $2 \mathrm{~cm}$ possam ser produtores, aqueles com mais de $3 \mathrm{~cm}$ apresentam maior probabilidade de serem hiperfuncionantes (11); o risco de evolução de um adenoma não produtor para produtor parece atingir um platô ao redor de 3 ou 4 anos de acompanhamento (11). Dados de várias séries pequenas indicam que aproximadamente $20 \%$ dos IA desenvolvem alterações bioquímicas quando acompanhados por 10 anos (1).

Os IA evoluem com aumento de volume em menos de $10 \%$ dos casos em 4 anos (2) e em até 30\% dos casos em 10 anos (1). É interessante ressaltar que em pacientes acompanhados por longos períodos a progressão para malignização é muito rara $(1,2)$.

Um estudo prospectivo (12) mostrou que o risco acumulado de um tumor não produtor passar a produzir cortisol era de $4 \%$ em um ano e 6,6\% em 5 anos; já o risco de um paciente com SC subclínica evoluir para SC clinicamente manifesta era de 12,5\% após um ano. Entretanto, outros estudos demonstraram que no seguimento dos IA a evolução para hiperfunção ocorreu em menos de $2 \%$ dos pacientes. A interpretação dos dados destas séries de seguimentos é prejudicada pela heterogeneidade dos períodos e das estratégias de acompanhamento adotados por cada uma delas. Desse modo, a evolução da SC subclínica parece ser benigna, raramente ocorrendo aumento da massa e evolução para a SC clinicamente manifesta $(<1 \%)$. Por esse motivo, a maioria dos autores não utiliza o termo "SC pré-clínica", e no Consenso publicado pelo NIH (1) concluiu-se que a melhor nomenclatura seria "hipersecreção glicocorticóide autônoma subclínica" (subclinical autonomous glucocorticoid bypersecretion). Desta forma, atualmente o termo "SC subclínica" tem sido amplamente utilizado (8).

Apesar da evolução aparentemente favorável, os incidentalomas e em especial a SC subclínica têm sido focos de estudos de diversos grupos. Isso em grande parte deve-se ao fato de que estes têm sido correlacionados de várias maneiras aos componentes da síndrome metabólica, em especial ao diabetes mellitus do tipo 2 . 
Pacientes com SC subclínica submetidos a remoção cirúrgica do tumor adrenal evoluem com perda de peso, e a redução dos níveis pressóricos e glicêmicos ocorre em mais de $70 \%$ dos indivíduos tratados.

\section{INCIDENTALOMAS DE ADRENAIS E SC SUBCLÍNICA X SÍNDROME METABÓLICA}

A elevada taxa de mortalidade observada na SC deve-se não apenas aos eventos cardiovasculares, mas também ao tempo de exposição ao hipercortisolismo (13-15).

A mortalidade na SC é 4 vezes maior do que na população geral, sendo que, em grande parte, esta é uma conseqüência da doença cardiovascular.

A hipertensão arterial sistêmica (HAS) e as alterações da glicemia estão entre as manifestações mais freqüentes da SC clássica, ocorrendo em até 90\% dos pacientes (16-20). Em IA, a HAS, o diabetes mellitus (DM) e a obesidade ocorrem em 41\%, 10\% e $28 \%$, respectivamente $(21)$. Esses achados são mais significativos se comparados à prevalência dessas doenças na população geral (22-25).

Os pacientes com incidentalomas, quando comparados a controles normais pareados para idade e índice de massa corpóreo (IMC), apresentam maiores níveis de pressão arterial (sistólica e diastólica) e de glicemia em teste clássico de sobrecarga oral de glicose, assim como menor sensibilidade à insulina (26).

O estudo de Rossi et al. (27) mostra uma alta prevalência de HAS, DM e dislipidemia em adenomas não produtores, mas principalmente naqueles com SC subclínica (tabela 1) (27).

Na SC subclínica, 76\% dos pacientes apresentam HAS, 33\%, DM tipo 2 e 50\%, obesidade (28).

Por essas razões, atualmente sugere-se que testes de triagem de SC devam ser realizados em pacientes com sinais e sintomas específicos e também naqueles que apresentam diagnósticos que podem ser resultado de excesso de cortisol (29).

Tabela 1. Comparação entre as manifestações clínicas em pacientes com adenomas adrenais não produtores e produtores de cortisol (27).

\begin{tabular}{lcc}
\hline & \multicolumn{2}{c}{ Adenoma Adrenal } \\
N & Não Produtor & SCS \\
IMC & 38 & 12 \\
Presença de: & $26,5 \pm 6,8$ & $25,6 \pm 5,9$ \\
HAS & & \\
DM2 & $34 \%$ & $92 \%$ \\
Dislipidemia & $26 \%$ & $50 \%$ \\
\hline
\end{tabular}

\section{SC SUBCLÍNICA E DIABETES MELLITUS}

Estudos realizados em pacientes com DM têm encontrado uma prevalência de SC subclínica igual ou maior do que a observada entre os incidentalomas de adrenais.

O estudo pioneiro de Leibowitz et al (30) avaliou 90 pacientes com IMC $>25 \mathrm{~kg} / \mathrm{m}^{2}, \mathrm{DM}$ e hemoglobina $(\mathrm{Hb})$ glicada superior a $9 \%$ (normal 4-8\%). Utilizou como triagem cortisol após $1 \mathrm{mg}$ de dexametasona, complementando a avaliação com $2 \mathrm{mg}$ por $72 \mathrm{~h}$ e com $8 \mathrm{mg}$, dosagem de cortisol urinário e de ACTH. Cinco pacientes apresentaram supressão alterada com $1 \mathrm{mg}$. Consideraram como hipercortisolismo autônomo a ausência de supressão com $2 \mathrm{mg}$, associada a cortisol urinário elevado. Foram realizados exames de imagem e o resultado anátomo-patológico confirmou os diagnósticos. Entre os pacientes estudados, encontraram um com adenoma adrenal e dois com doença de Cushing (DC), totalizando 3,3\% de SC. Após as respectivas cirurgias, houve normalização dos níveis de cortisol urinário e do teste de supressão com dexametasona, assim como redução da $\mathrm{Hb}$ glicada (figura 2) (30).

Posteriormente, Catargi et al (31) avaliaram 200 pacientes com DM2, IMC $\geq 25 \mathrm{~kg} / \mathrm{m}^{2}$ e Hb glicada $>8 \%$ (normal: $6 \%$ ). Realizaram a triagem com 1 $\mathrm{mg}$ de dexametasona, prosseguindo a avaliação com 4 mg de dexametasona endovenosa, ritmo de cortisol, dosagem de ACTH e cortisol urinário de $24 \mathrm{~h}$, ressonância magnética de hipófise e/ou tomografia de adrenais e cintilografia de adrenais com iodocolesterol. Nesta série encontraram-se $15 \%$ de resultados falsopositivos na triagem: $8,5 \%$ dos pacientes tiveram 2 testes alterados, $5,5 \%$ dos estudados tinham imagem alterada ( $1,5 \%$ com adenoma hipofisário e $4 \% \mathrm{com}$ tumor adrenal).

Onze pacientes apresentavam alterações bioquímicas associadas a exames de imagem alterados. Foi considerado como diagnóstico definitivo os pacientes encaminhados para cirurgia e com resultado anátomopatológico confirmatório. Quatro pacientes preenchiam os critérios ( 3 com DC e l com adenoma adrenal), totalizando $2 \%$ de SC da série inicial. Seis meses após o tratamento da SC, houve redução média de $5,5 \%$ do peso corporal e de $2,5 \%$ da $\mathrm{Hb}$ glicada (31).

Chiodini et al (32) avaliaram 294 pacientes com DM2 e 189 controles, com IMC $\geq 19$ e $<50$ $\mathrm{kg} / \mathrm{m}^{2}$. Realizou-se teste de supressão com $1 \mathrm{mg}$ de dexametasona e, nos que apresentaram resultados alterados, o teste foi repetido. A seguir, realizaram dosagens de cortisol urinário de $24 \mathrm{~h}, \mathrm{ACTH}$, cortisol plasmático às $23 \mathrm{~h}$ e cortisol plasmático após dexa- 

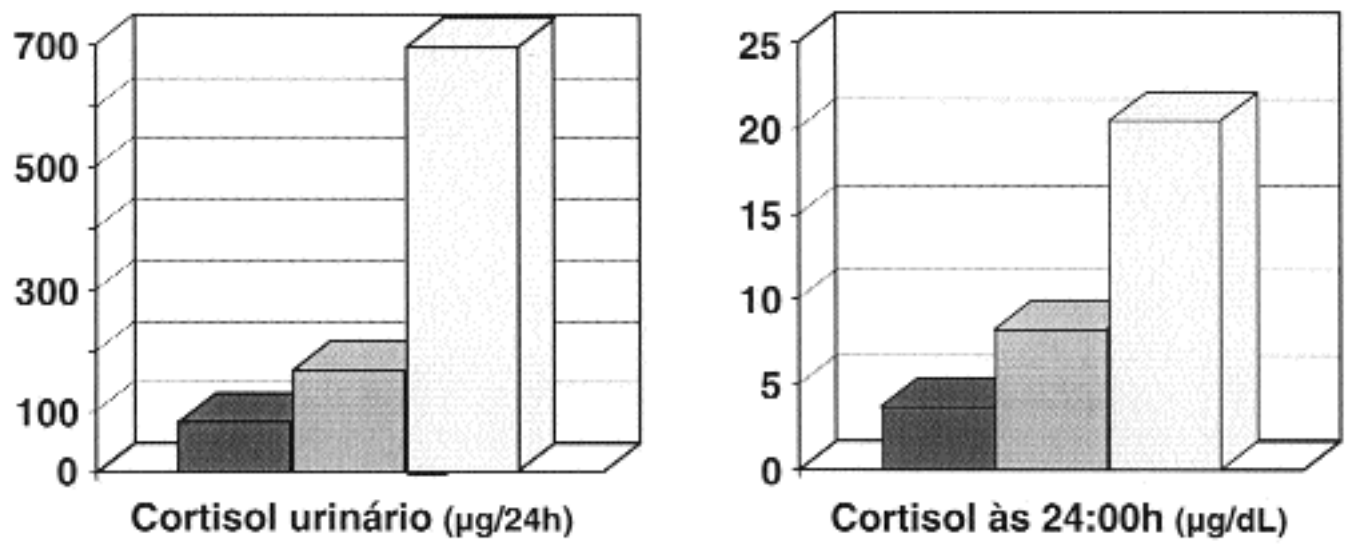

\section{Não Funcionantes $\square$ S.Cushing Subclínica $\square$ S.Cushing Manifesta}

Figura 2. Niveis de cortisol urinário e cortisol sérico à meia-noite em pacientes com tumor adrenal não-funcionante, síndrome de Cushing (SC) subclínica e SC manifesta (26).

Tabela 2. Resultados do teste de triagem e dos exames de imagem em pacientes com diabetes mellitus do tipo 2 (DM2) e controles (32).

\begin{tabular}{lcc}
\hline & DM2 & Controles \\
\hline Falso-positivos (Dex lmg): & $5,4 \%$ & $3,1 \%$ \\
Tumor adrenal $(\mathrm{n}):$ & 21 & 3 \\
Tumor hipófise $(\mathrm{n}):$ & 4 & 1 \\
Massa pulmonar $(\mathrm{n}):$ & 2 & 0 \\
\hline
\end{tabular}

Tabela 3. Comparação entre os estudos que investigaram SC em pacientes com DM.

\begin{tabular}{|c|c|c|c|c|}
\hline & Lebowitz (30) & Catargi (31) & Chiodini (32) & Caetano (33) \\
\hline Idade (anos) & $53,5 \pm 1,6$ & $58,6 \pm 10,7$ & $60,9 \pm 10,2$ & $56,4 \pm 8,8$ \\
\hline$M / F$ & $26 / 64$ & $49 / 151$ & $124 / 165$ & $34 / 69$ \\
\hline \multicolumn{5}{|l|}{ IMC (kg/m²) } \\
\hline $\mathrm{M}$ & $31,0 \pm 1,0$ & $32,7 \pm 5,5$ & $29,2 \pm 4,3$ & $30,5 \pm 5,1$ \\
\hline $\mathrm{F}$ & $33,9 \pm 0,8$ & $34,4 \pm 6,7$ & $31,7 \pm 5,6$ & $31,7 \pm 4,6$ \\
\hline \multicolumn{5}{|l|}{ RCQ } \\
\hline M & NA & $1 \pm 0,1$ & NA & $0,99 \pm 0,05$ \\
\hline $\mathrm{F}$ & NA & $0,9 \pm 0,1$ & NA & $0,95 \pm 0,07$ \\
\hline Hb glicada (\%) & $12,2 \pm 0,3$ & $10,8 \pm 1,8$ & $9,6 \pm 2,4$ & $8,6 \pm 2,2$ \\
\hline Cortisol após Dex 5 mg & $5 \mu \mathrm{g} / \mathrm{dL}$ & NA & NA & NA \\
\hline Cortisol após Dex 1 mg & NA & $2,1 \mu \mathrm{g} / \mathrm{dL}$ & $1,8 \mu \mathrm{g} / \mathrm{dL}$ & $1,8 \mu \mathrm{g} / \mathrm{dL}$ \\
\hline Falso-positivo pós-Dex (Triagem) & $1,1 \%$ & $15 \%$ & $5,4 \%$ & $13,7 \%$ \\
\hline Relação Cortisol x IMC & Aus & Aus & NA & Aus \\
\hline Relação Cortisol x HbAlc & Aus & Aus & NA & Aus \\
\hline
\end{tabular}

metasona, além de estímulo com CRH e exames de imagens. Os resultados estão mostrados na tabela 3. Neste estudo, o diagnóstico de hipercortisolismo com exames de imagem compatíveis foi de $7 \%$ da série inicial. Assim como nos anteriores os pacientes que foram submetidos a tratamento da SC apresentaram melhora no controle do DM (32).

Recentemente (33), nosso grupo avaliou 103 pacientes com DM2, IMC $\geq 25 \mathrm{~kg} / \mathrm{m}^{2}$, utilizando como triagem o cortisol salivar às $23 \mathrm{~h} \mathrm{e} \mathrm{o} \mathrm{cortisol} \mathrm{sali-}$ var e plasmático após $1 \mathrm{mg}$ de dexametasona. Realiza- mos supressão com $2 \mathrm{mg}$ de DEX por $48 \mathrm{~h}$, cortisol urinário de 24h, ACTH, SDHEA, cortisol e ACTH após DDAVP, ressonância magnética de hipófise e tomografia computadorizada de adrenais. Oito pacientes apresentavam 2 ou mais testes positivos, sendo que 3 dos pacientes apresentavam alta probabilidade de diagnóstico de SC subclínica, em concordância com os resultados de Leibowitz (30) e Catargi (31).

Nenhum dos estudos encontrou correlação entre as alterações do eixo HHA e os controles de glicemia (tabela 3). 


\section{CONCLUSÃO}

A SC subclínica segue como um desafio desde o diagnóstico até o tratamento. Ainda não se estabeleceu a melhor abordagem diagnóstica, sendo que a maioria dos autores tende a utilizar a supressão com $1 \mathrm{mg}$ de dexametasona como teste de triagem.

A literatura atual sugere que os incidentalomas de adrenais estão relacionados às características clínicas da síndrome metabólica, embora ainda não se possa descartar que, em alguns casos, eles sejam parte das manifestações da síndrome e não a causa deste complexo metabólico. Os estudos disponíveis demonstram que em parte dos pacientes ocorre controle da pressão arterial e da glicemia após tratamento da SC subclínica; entretanto, o número de pacientes avaliados ainda é pequeno. Além disso, não há evidências de que os pacientes tratados terão benefícios a longo prazo. Do mesmo modo, não há consenso sobre quando indicar cirurgia e, se optar-se por tratamento clínico, como será feito o acompanhamento. Nos pacientes assintomáticos (sem HAS e DM) deve-se considerar o tratamento cirúrgico, se além da ausência de supressão houver também elevação do cortisol urinário e, no caso de tumor adrenal, o ACTH estiver suprimido (28).

As evidências atuais indicam que há benefícios para os pacientes tratados para a SC subclínica; no entanto, o número de indivíduos avaliados e o tempo de seguimento ainda são insuficientes para que se possa definir qual a melhor abordagem nesses casos. A tabela 4 confronta os argumentos para tratar-se ou não a SC subclínica.

As variações entre os estudos quanto ao diagnóstico, critérios de tratamento e formas de seguimento dos pacientes são os maiores limitantes para que se possa estabelecer a melhor abordagem para os pacientes com SC subclínica, quer diagnosticados devido a incidentaloma de adrenal ou na triagem de indivíduos com DM, sendo que, nestes, os dados são ainda mais escassos do que nos incidentalomas.

Tabela 4. Confronto entre os argumentos para tratar ou não a SC subclínica.

\section{Porque Tratar}

Pode levar a perda de peso e melhora dos níveis de pressão arterial, glicemia e lípides Pode reduzir o risco cardiovascular

Previne a progressão para a SC clínica

\section{Porque Não Tratar}

Há poucos pacientes avaliados e os estudos não são homogêneos

Não há estudos de longo prazo comprovando essa redução

A progressão em não tratados é discutível
Além das séries com pacientes portadores de DM2, a prevalência da SC subclínica ou mesmo clínica, deve estar possivelmente aumentada em outras populações de pacientes em risco, como portadores de hipertensão arterial, dislipidemia e naqueles com osteoporose ou osteopenia. Maiores estudos, englobando grande número de pacientes e tempo adequado de seguimento, mostram-se necessários para documentar essa possibilidade.

\section{REFERÊNCIAS}

1. NIH State-of-the-Science Statement on management of the clinically inapparent adrenal mass ("incidentaloma"). NIH Consens State Sci Statements 2002;19(2):1-23.

2. Barzon L, Sonino N, Fallo F, Palu G, Boscaro M. Prevalence and natural history of adrenal incidentalomas. Eur $\mathbf{J}$ Endocrinol 2003;149:273-85.

3. Ross NS. Epidemiology of Cushing's syndrome and subclinical disease. Endocrinol Metab Clin N Am 1994;23:539-46.

4. Boscaro M, Barzon L, Fallo F, Sonino N. Cushing's syndrome. Lancet 2001;357:783-91.

5. Arnaldi G, Angeli A, Atkinson AB, Bertagna X, Cavagnini $F$, Chrousos GP, et al. Diagnosis and complications of Cushing's syndrome: a consensus statement. J Clin Endocrinol Metab 2003;88:5593-602.

6. Findling JW, Raff H. Clinical review: Cushing's syndrome. J Clin Endocrinol Metab 2006;91(10):3746-53.

7. Newell-Price J, Trainer P, Besser M, Grossman A. The diagnosis and differential diagnosis of Cushing's syndrome and pseudo-Cushing's states. Endocr Rev 1998;19:647-72.

8. Terzolo M, Bovio S, Reimondo G, Pia Anna, Osella G, Borretta $G$, et al. Subclinical Cushing's syndrome in adrenal incidentalomas. Endocrinol Metab Clin North Am 2005;34:423-39.

9. Young WF Jr. The incidentally discovered adrenal mass. $\mathbf{N}$ Engl J Med 2007;356(6):601-10.

10. Mansmann G, Lau J, Balk E, Rothberg M, Miyachi $Y$, Bornstein SR. The clinically inapparent adrenal mass: Update in diagnosis and management. Endocr Rev 2004;25(2):309-40.

11. Terzolo M, Reimondo G, Bovio S, Angeli A. Subclinical Cushing's syndrome. Pituitary 2004;7:217-23.

12. Barzon L, Fallo F, Sonino N, Boscaro M. Development of overt Cushing's syndrome in patients with adrenal incidentaloma. Eur J Endocrinol 2002; 146:61-6.

13. Mantero F, Boscaro M. Glucocorticoid-dependent hypertension. J Steroid Biochem Mol Biol 1992;43(5):409-13.

14. Arnaldi G, Masini AM, Giacchetti G, Taccaliti A, Faloia E, Mantero F. Adrenal incidentaloma. Braz J Med Biol Res 2000;33(10):1177-89.

15. Dekkers OM, Biermasz NR, Pereira AM, Roelfsema F, van Aken MO, Voormolen JHC, et al. Mortality in patients treated for Cushing's disease is increased, compared with patients treated for nonfunctioning pituitary macroadenoma. J Clin Endocrinol Metab 2007;92:976-81.

16. Ross EJ, Linch DC. Cushing's syndrome-killing disease: discriminatory value of signs and symptoms aiding early diagnosis. Lancet 1982;2:646-9.

17. Urbanic RC, George JM. Cushing's disease -18 years' experience. Medicine (Baltimore) 1981;60:14-24.

18. Soffer LJ, lannaccone A, Gabrilove JL. Cushing's syndrome: A study of fifty patients. Am J Med 1961;300:129-35.

19. Sprague RG, Randall RV, Salassa RM, Scholz DA, Priestley JT, Walters W, et al. Cushing's syndrome: A progressive and often fatal disease. Arch Intern Med 1956;98:389-98.

20. Plotz CM, Knowlton Al, Ragan C. The natural history of Cushing's syndrome. Am J Med 1956;13:597-614. 
21. Mantero F, Terzolo M, Arnaldi G, Osella G, Masini AM, Ali A, et al. A survey on adrenal incidentaloma in Italy. J Clin Endocrinol Metab 2000;85(2):637-44.

22. Malerbi DA, Franco LJ. Multicenter study of the prevalence of diabetes mellitus and impaired glucose tolerance in the urban Brazilian population aged 30-69 yr. Diabetes Care 1992;15(11):1509-16.

23. King $H$, Aubert RE, Herman WH. Global burden of diabetes, 1995-2025. Diabetes Care 1998;21(9):1414-31.

24. Winer N, Sowers JR. Epidemiology of diabetes. J Clin Pharmacol 2004;44:397-405.

25. Laaksonen DE, Niskanen L, Lakka HM. Epidemiology and treatment of the metabolic syndrome. Ann Med 2004;36:332-46.

26. Terzolo M, Pia A, Ali A, Osella G, Bovio S, Angeli A, et al. Adrenal incidentaloma: a new cause of the metabolic syndrome? J Clin Endocrinol Metab 2002;87(3):998-1003.

27. Rossi R, Tauchmanova L, Luciano A, Martino MD, Nuzzo V, Lombardi G, et al. Subclinical Cushing's syndrome in patients with adrenal incidentalomas: clinical and biochemical features. J Clin Endocrinol Metab 2000;85(4):1440-8.

28. Sippel R, Chen H. Subclinical Cushing's syndrome. Surg Clin North Am 2004;84:875-85.

29. Findling JW, Raff H. Screening and diagnosis of Cushing's syndrome. Endocrinol Metab Clin North Am 2005;34: 385-402.
30. Leibowitz G, Tsur A, Chayen SD, Salameh M, Raz I, Cerasi E, et al. Pre-clinical Cushing's syndrome: an unexpected frequent cause of poor glycaemic control in obese diabetic patients. Clin Endocrinol (Oxf) 1996:44:717-22.

31. Catargi B, Rigalleau V, Poussin A, Ronci-Chaix N, Bex V, Tabarin A, et al. Occult Cushing's syndrome in type-2 diabetes. J Clin Endocrinol Metab 2003;88:5808-13.

32. Chiodini I, Torlontano M, Scillitani A, Arosio M, Bacci S, Di Lembo S, et al. Association of subclinical hypercortisolism with type 2 diabetes mellitus: a case-control study in hospitalized patients. Eur J Endocrinol 2005;153:837-44.

33. Caetano MSS, Silva RC, Kater CE. Increased diagnostic probability of subclinical Cushing's syndrome in a population sample of overweight adult patients with type 2 diabetes mellitus. Arq Bras Endocrinol Metab 2007;51/7:1118-27.

\section{Endereço para correspondência:}

Claudio E. Kater

Professor Associado de Medicina

Divisão de Endocrinologia, Departamento de Medicina

Universidade Federal de São Paulo

Rua Pedro de Toledo 781, 13 andar

04039-032 São Paulo, SP

E-mail: kater@unifesp.br 